九州大学学術情報リポジトリ

Kyushu University Institutional Repository

Ignorance as a Limitation for the Application of Scientific Methods to Environmental Protection Activities

Watanabe, Takashi

Green Asia Education Center, Kyushu University : Assistant Professor

https://doi.org/10.5109/1500426

出版情報: Evergreen. 2 (1)，pp.41-48，2015-03. Green Asia Education Center バージョン：

権利関係 : 


\title{
Ignorance as a Limitation for the Application of Scientific Methods to Environmental Protection Activities
}

\author{
Takashi Watanabe* \\ Green Asia Education Center, Kyushu University, Japan \\ *Author to whom correspondence should be addressed, \\ E-mail: watanabe.takashi.280@m.kyushu-u.ac.jp
}

(Received January 14, 2015; accepted February 23, 2015)

\begin{abstract}
In the field of science and technology, an open discussion of ignorance as a fundamental cause of environmental destruction has long been avoided. However, this does not mean that ignorance has become obsolete or irrelevant altogether because it has yet been well discussed in the field of philosophy. The different attitudes toward ignorance between the fields of science and philosophy imply that it is a slippery and almost inconceivable object for science, whereas it is something essential to grasp and examine for philosophy. To understand its slippery nature, therefore, we suggest that ignorance is best examined from an epistemological viewpoint, as something that oscillates between reality and nonreality that appears on the surface and then hides underground. In this paper, we explore how ignorance escapes from the scientist's careful observation and affects the results of actual comprehensive environmental protection measures such as the precautionary principle and risk assessment.
\end{abstract}

Keywords: risk assessment, precautionary principle, uncertainty, ignorance, catastrophe principle

\section{Introduction}

It is obvious that human beings are responsible for protecting and preserving the global environment on which all life, including our own, depends. However, it is not so easy as we might imagine to identify the necessary measures that are based on the scientific knowledge now available with which to fulfill those responsibilities. Admittedly, we already have two major measures for protecting the environment in the form of risk assessment (RA) and the precautionary principle (PP), but they seem to have certain flaws due to scientific uncertainty that might lead to a result that is the opposite of what was initially intended. For example, (1) scientific uncertainty may cause unknown effects in the environment when certain technology is implemented to protect it, (2) the degree of impact of these unknown effects can result in the extinction of all living things including human beings, and (3) it is almost impossible to eliminate scientific uncertainty no matter how science/technology will advance in the future.

A crucial aspect of these flaws is that some of them seem to occur due to ignorance, which is known as an epistemological blind spot.

Ignorance, I argue hereafter, is generally considered as a sort of scientific uncertainty, which is the main reason for taking a precautionary approach. However, unlike scientific uncertainty, which is commonly discussed in the field of science, when ignorance is concerned, any precautionary approach on the basis of scientific knowledge can become useless ${ }^{1)}$ except in its extreme form, such as completely suspending the application of science/technology in any form whatsoever. We will see later that a situation like this inevitably results when science/technology is applied to the environment.

An argument concerning ignorance often emerges in connection with the idea behind the PP in the field of environmental philosophy. However, in most cases the difference between scientific uncertainty and ignorance remains unclear, and hence the impact of ignorance is underestimated.

To understand this vague idea, therefore, we first describe the epistemic standards for environmental hazards, and carefully define and focus on ignorance as a cause of unexpected consequences, including catastrophes.

Second, by examining the general arguments that defend the PP, we elucidate the incompetency of actions taken on a scientific basis to treat or curb environmental degradation when ignorance is seriously considered.

Finally, we demonstrate that the problem discussed here should not be reduced to that of decreasing or mitigating the risk of each technological product as has been commonly implemented with risk evaluation and cost-benefit analysis (CBA). Rather, we mention that investigation of the problem should be further developed into inquiries concerning whether the utilization of science/technology itself can be essential for addressing environmental degradation, and hence whether we ought to force ourselves to consider the comprehensive prevention of the use of science/technology itself to 
conduct valid environmental protection measures.

Usually, this type of argument does not draw people's attention and is mostly ignored as an unrealistic zero-sum game. People tend to take for granted that practical protection measures should be based on certain scientific knowledge; for example, ongoing general environmental protection measures, such as saving energy, reducing greenhouse gas emission, implementing regulations for creating and providing genetically engineered organisms, are all supposed to be based on scientific knowledge. If they do not have any scientific basis, they cannot apply to any practical problems. However, this article questions the authority of science itself on which all those protection measures depend. Needless to say, this article may easily arouse potential criticism, for it ruins the grounds for the validity of practical environmental protection measures as a whole.

Nevertheless, if this paper succeeds in describing the unique nature of environmental problems, and is persuasive enough to force us to accept it as a practical issue, we must take a certain course of action to solve it, however preposterous the action might seem to us. Therefore, what is the point of still insisting on current protection measures when the grounds of their validity have been appraised to be less reliable than we once thought?

\section{The Precautionary Principle and Ignorance}

Versions of the PP appear in a number of international treaties and regional policy statements such as the UN Framework Convention on Climate Change (1992), the Ozone Layer Protocol (1987), and the US President's Council on Sustainable Development (1996). In reviewing many statements of the PP, it can be observed that they share the same general structure. For example, the basic idea of the PP can be seen in one widely cited statement:

When an activity raises threats of harm to human health or the environment, precautionary measures should be taken even if some cause and effect relationships are not fully established scientifically. ${ }^{2)}$

A notable feature of the PP is that it publicly addresses the problem of uncertainty, especially that of ignorance, which extends beyond science and cognition ${ }^{3)}$. This can result in confusion and certain paradoxes, such as the catastrophe principle (CP), which permits the avoidance of not only any action but also its contradictory. However, if we insist on applying the PP by restricting it to the scope of scientific and cognitive to avoid this inconvenience ${ }^{4)}$, we will overlook the problem of ignorance.

\section{Classification of Danger}

The PP emerged as a step toward protecting and restoring ecosystems that would supplement RA and CBA. Critics have largely attacked the latter two practices because they depend on the presupposition that humans are capable of completely understanding the impact of their activities on the environment and addressing the harm caused to the environment and humans. ${ }^{5)}$ In fact, it is evident that there is no clear basis for supporting this presupposition. For example, when we classify environmental hazards from an epistemological viewpoint, we can easily identify certain situations that may escape scientists' careful observations.

The epistemic standards for environmental hazards are generally classified into three groups:

1. Risk: "all possible outcomes are known in advance and ... their relative likelihood can be adequately expressed as probabilities."

2. Uncertainty: "the adequate empirical or theoretical basis for assigning probabilities to outcomes does not exist."

3. Ignorance: "not only ... a lack of certainty as to the likelihood of different outcomes, but ... some of the possibilities themselves remain unknown." 6)

RA and CBA apply only to the first group because the circumstance of risk stipulates that the probability information is known ${ }^{7)}$. The PP applies to the others, wherein the cause and effect relationships are not fully established scientifically. However, the PP still has neither a commonly accepted definition nor a set of criteria in these circumstances to guide its implementation. This creates some problems as outlined below:

A) The PP lacks a specific definition to prevent it from being arbitrarily used. For example, if the burden of proof were shifted from the public (governments and nongovernmental organizations) to industry and the scientific community, a consistent application of the PP would stifle all innovation. ${ }^{8)}$

B) The PP might lead to the neglect of other dangers when it is being applied to a specific danger. For example, in Japan the cumulative risk of cancer that is attributable to diagnostic $\mathrm{X}$-rays could be more than $3 \%$, which is equivalent to approximately 7,500 cases of cancer per year.9) However, the calculations involve a number of assumptions, and so are inevitably subject to considerable uncertainty. We have no data indicating that cancer is actually attributable to diagnostic X-rays. ${ }^{10)}$ Therefore, if we applied the PP to this problem, 
we would have to restrict the frequent use of diagnostic X-rays to prevent uncertain outcomes such as losing the benefit of early-stage detection and treatment of diseases. In such cases wherein the risk-return trade-off occurs, the PP would not function well.

C) The application of the PP itself might cause new dangers. If so, then the application of the PP should be prevented. ${ }^{11}$ )

For example, Comstock claims that, as the proponents of the PP maintain, developing genetically modified (GM) crops might lead to environmental degradation. However, if we ban GM crops based on the PP, global food shortages caused by global warming might follow. If so, then we must develop GM crops, according to the PP. ${ }^{12)}$ In reference to this, Sunstein says, "The regulation that the principle requires always gives rise to risks of its own - and hence the principle bans what it simultaneously mandates."

Considering these problems, Manson argues that regulations and prohibitions warranted by the PP should be limited to those factors that are less damaging to the environment than commercial and technological development. ${ }^{14)}$ It seems that for Manson, the PP represents nothing more than a cause of environmental degradation. In addition, he claims that implementing CBA between the value of a functioning ecosystem and of industrial/technological development can be more appropriate and useful for decision making than the PP because the value of an ecosystem is so high as to be almost beyond calculation, whereas the value of industrial/technological development seems low in comparison:

For the tropical rainforests as a whole, the cost of replacing such services with our own would far exceed the gross international product. Similarly, entities such as lakes, fish populations, and coral reefs can be viewed as "natural capital" into which it is unwise (according to conventional economic wisdom) to dip. ... Rather than hesitate to assign monetary value to environmental objects and processes, environmentalists should hasten to do so. They will, I think, be pleasantly surprised by how often cost-benefit analyses rule in their favor. ${ }^{15}$ )

Is Manson's claim appropriate? Should we implement CBA instead of the PP as Manson says? If so, then how should we deal with the problem of uncertainty and ignorance? As we have just seen, CBA is useless for those two epistemological situations. We therefore still need the PP to address those situations, though they are actually the cause of the defect in the PP. We must find an appropriate way to operate the PP. Therefore, the most serious problem among the three shown above is that of
(C), which we examine in the next chapter.

\section{General Arguments Defending the Precautionary Principle}

The paradox derived from the application of the PP stems particularly from the state of ignorance wherein the PP is to be applied. Ignorance is a state of not knowing how unpleasant surprises spring up. In such cases, as we have already seen, even applying the PP might cause unexpected results that lead to the banning of the application of the PP itself. Furthermore, such reasoning fits almost every technology and what it produces. For example, Manson argues the following:

[I]t could be that preventing global warming has some nonzero probability of leading to nuclear winter. Significant reductions in the consumption of fossil fuels might cause global economic collapse, which might cause global war, which might lead to multiple detonations of nuclear weapons, which might lead to a nuclear winter. If the Catastrophe Argument for drastic reductions in the emission of greenhouse gasses is a good one, then, by parity of reasoning, we have an equally good argument against drastic reductions in the emission of greenhouse gasses. ${ }^{15)}$

Here, Manson implies that by stretching our imagination to the utmost limit, the catastrophic results can easily be made to authorize the prevention of any action and its contradictory. This is what he calls the catastrophe principle. Once ignorance is considered, as it allows any imaginable cause-relationship, we cannot but introduce any figment of our imagination to illustrate catastrophic consequences. However, is this line of thought appropriate?

Gardiner says that the example Manson puts on the table is logically possible but not realistic, that the PP can be properly applied so far as the range of outcomes considered represents credible threats supported by certain scientific reasons:

$[\mathrm{P}]$ roponents of precaution usually demand some kind of scientific reason for thinking that there may be a problem. So they envisage natural possibilities. (The standard cases involve scientific evidence for the threat. For example, in climate change, there is scientific knowledge of the basic greenhouse mechanism, together with a body of empirical evidence; with genetically-modified foods, there is some previous experience with newly introduced species.) $)^{16)}$

This brings our argument to a claim, as Gardiner suggests, that the concrete situations to which the PP is applied should be limited to that of uncertainty, which requires some explicit or implicit criteria for realistic outcomes. ${ }^{17)}$ Here, the state of ignorance is literally 
ignored.

Saner goes further by proposing a "second order risk evaluation methodology" as an answer to avoiding the problem of the $\mathrm{CP}$ wherein both a precautionary action and inaction lead to the conditions that trigger the PP. According to him, applying risk evaluation (RE) to a precautionary action is useful in finding "the location of risk neutrality where more stringent precaution becomes counter-productive." ${ }^{18)}$ This means that RE contributes to the application of the PP in a rational manner.

Thus, we can make the room for the proper use of the PP by excluding the state of ignorance wherein any imaginable outcome happens and by dealing only with a state of scientific uncertainty wherein all possible outcomes are previously known scientifically.

Practically speaking, RE is now commonly employed as a complement to the PP; the application of the PP is accepted as valid only when accompanied by the RE, on the basis of an examination of potential benefits and costs of action. For example, in the "Twelve Late Lessons" proposed by the European Environmental Agency, we can see related issues such as "Identify and work to reduce 'blind spots' and gaps in scientific knowledge," "Systematically scrutinize the claimed justifications and benefits alongside the potential risks," and "Evaluate a range of alternative options for meeting needs alongside the option under appraisal, and promote more robust, diverse and adaptable technologies so as to minimize the costs of surprises and maximize the benefits of innovation."')

Thus the validity of the PP is secured, and thereby the state of ignorance is overlooked even in the most practically promising environmental protection activities. However, the PP should have been originally established to deal with ignorance- a situation wherein RE and CBA are both useless. Then is it not still necessary to consider situations that stem from ignorance, and to which it is impossible to apply both RE and CBA?

\section{The Problem of General Arguments Defending the Precautionary Principle}

The general arguments that support the PP, as shown above, have some problems as follows:

(i) The PP's relying upon RE and CBA cannot avoid the CP for, as mentioned earlier, the PP in this context inevitably gives rise to unexpected catastrophic outcomes that stem from a state of ignorance. The work of specifying and reducing blind spots and gaps in scientific knowledge is therefore insufficient to eliminate the occurrence of unexpected outcomes. However, promoting "more robust, diverse and adaptable technologies so as to minimize the costs of surprises and maximize the benefits of innovation"6) would increase the possibility of the occurrence of unexpected outcomes.

(ii) Basically, the PP that is based on RE is not supposed to aim at zero risk, but rather lower or more acceptable risks or hazards via scientific research. ${ }^{19)}$ This suggests that the application of the PP in such conditions requires taking advantage of scientific knowledge to determine risks or hazards. Here, the need for science/technology is a matter of course from the beginning; there is no suggestion of weeding it out at all. However, as we expand the development and use of science/technology, we will naturally be faced with an increasing number and degree of catastrophic outcomes, some of which may stem from a state of ignorance.

Understood in this way, defending the PP without considering the problem of ignorance is an inadequate strategy. Further examination of ignorance is required to protect the PP from the CP.

\section{Classification of Ignorance}

As mentioned, the epistemic standards for environmental hazards are generally classified into three groups: risk, uncertainty, and ignorance. This classification stems from scientific methods such as measurement, observation, experiment, and theorization, and only phenomena captured by these methods are categorized as scientific knowledge. Thus, there should be a particular domain of something located outside the jurisdiction of science from which the hazards classified as uncertainty and ignorance arise. We illustrate the actual events of uncertainty and ignorance below:

\section{Uncertainty:}

1. Unexpected situations generated from a multiplicity of interactions of a system's components, which sometimes results in a new, irreversible system:

Chaos theory and the theory of black swan events are included in this category, for example. ${ }^{20) 21)}$ In these cases, unexpected surprises arise on account of the combination of a finite number of components of a system. The results can be described as, for example, price, wage, or production indexes in economics. Therefore, although a forecast of the outcome is difficult, the outcome itself is understandable, and its cause-effect relations are identifiable. ${ }^{22}$

2. Epistemologically recognizable outcomes of which the correlation and causation among the components involved are not identifiable:

When we examine types of phenomena like hay fever, atopic dermatitis, El Niño/La Niña-Southern Oscillation, and mass strandings of dolphins and whales, for example, it is possible to observe and recognize what actually happened, but mainly it is difficult or impossible to know why and how these events occurred. This is because there are some cases wherein it is possible to establish more than one hypothesis based on thorough experiments concerning an identical phenomenon, but not possible to decide which hypothesis is relevant. 


\section{Ignorance:}

3. Situations wherein it is impossible to identify their cause-effect relations and to know what actually happened:

There might exist certain situations wherein it is impossible to recognize and identify the same phenomenon and therefore impossible to experimentally replicate it. Hence, scientific methods will not be appropriate in dealing with these sorts of situations. For example, (a) cases wherein undiscovered and hence unknown new species become extinct, (b) cases wherein the expected emergence and evolution of new species are eliminated in advance, (c) cases wherein a malfunction of the brain, for example, causes the retrogression or degradation of the intelligence of all humanity, which leads to a disturbance in recognizing and identifying the same phenomenon.

\section{Outside of both perceivable and imaginable} situations that include the situations illustrated above:

This type of ignorance cannot be the object of thought because it is neither perceivable nor imaginable. Walker et al. suggest that ignorance itself is unknown: "We have no way of knowing the full extent of our ignorance."23)

When considering the classification of uncertainty and ignorance shown above, we face another problem: How is it possible for us to know when unexpected outcomes occur that are included in the state of ignorance shown in cases 3 and 4? Is it possible to identify and indicate outcomes that are not imaginable and hence cannot be the objects of thought? Is it not an erroneous assumption to interpret those that cannot be stated situations as situations? Furthermore, is it not contradictory and inappropriate to assert that industrial/technological development generates unexpected outcomes, when the correlation and causation among the components involved cannot be identified?

The validity of the assertion concerned with environmental hazards can be justified in circumstances wherein the relationship between unexpected outcomes and industrial/technological development is established. However, the situations shown in cases 3 and 4 do not fulfill this condition. Therefore, is it not logically invalid to include those states of ignorance in our discussion at all?

It exceeds human competence to hold that we can know all the things in the world that are hidden from human intelligence. If the entire world is not comprised of phenomena that are perceivable and scientifically understandable for human beings, then we must be able to assume that certain relations are unidentifiable through existing scientific methods and measures and that they can be either relations that are resolved by further scientific development and research or relations that are irreducible to scientific knowledge at all.

\section{Refutation of the Catastrophe Principle}

Based on careful consideration of the arguments concerning ignorance stated above, we now examine the validity of the PP. As Comstock suggests in his argument about GM crops or Manson in his catastrophe argument, if the application of the PP actually generates unexpected outcomes, such as the $\mathrm{CP}$ indicates, it seems relevant to prevent the application of the PP itself.

However, the argument that presupposes unexpected outcomes, they suggest, seems unacceptable for the following reasons:

(a) The argument combines the idea of unexpected outcomes with the PP by employing very likely situations such as the credible threats or threats that are identified as unexpected. However, in those situations, either their cause-effect relations are scientifically specifiable or they are to be included in the level of uncertainty (outcome known, but its probability not calculable due to the lack of a sufficient empirical or theoretical basis); therefore, unexpected outcomes classified as ignorance are not considered.

(b) If we consider ignorance, then our main concern, expressed as a proposition below, brings us to a further problem:

If the application of the PP itself might cause a new catastrophic danger, then the application of the PP should be prevented.

The antecedent of this proposition contains the concept of unexpected outcome, which is expressed as catastrophic danger. If this concept implies the outcomes categorized within the state of ignorance, as shown in cases 3 and 4, then the antecedent of the proposition asserts neither what nor how the application of the PP will cause catastrophic damage in the future. In other words, the unexpected outcome has no extension. Furthermore, if ignorance is taken into account, the causal relations between the application of the PP and unexpected outcomes cannot be established. Given all this, then the antecedent of the proposition is false, and the truth-value of a proposition that has a false antecedent is always true. As a result, the CP becomes invalid so far as the state of ignorance is concerned.

If that is the case, then we can scarcely admit the criticism against the PP that is suggested by Manson and other researchers insofar as unexpected outcomes ranging from risk to uncertainty are concerned, for the $\mathrm{CP}$ is logically valid in this area. However, when we take ignorance into consideration, the criticism against the PP no longer has validity.

This argument entails another difficult problem: under the circumstance of ignorance, the propositions shown below are also both true. 
1. If the application of the PP might cause a new catastrophic danger, then the PP should be applied.

2. If the utilization of scientific knowledge might cause a new catastrophic danger, then scientific knowledge should be utilized.

These examples might suggest that propositions are logically useless when their antecedents include the term ignorance, and that we must restrict our argument concerning unexpected outcomes to the categories ranging from risk to uncertainty. However, as we have mentioned, this restriction weakens the PP. We need a PP that can deal with ignorance by avoiding the paradox derived from the CP.

To support the claim that the PP is applicable to ignorance is, however, as mentioned, to deny the causal relation between the utilization of science/technology and environmental degradation. When there is no perceptible event as an effect, we cannot identify either its cause or the relation between them. Nevertheless, insofar as we regard ignorance as an irreducible domain from which unexpected outcomes may arise, we need to admit that there should be a certain kind of unknown connection between unexpected outcomes and the utilization of science/technology, even if it leads us to a logical invalidity.

Because there is no logical meaning, should we reject such an argument?

\section{The Knowledge and Understanding Derived from Experience}

Where ignorance is concerned, the lack of a perceptible causal relation between the utilization of science/technology and environmental degradation does not necessarily mean that there is no causal relation between them at all. The reason why we do not recognize the relation in this case is that we interpret it through scientific methods such as observation, experiment, measurement, and theorization. In other words, these scientific methods reveal all but certain causal relations that stem from the state of ignorance, which is classified as a domain beyond the scope of the scientific and cognitive. $^{24)}$

If this line of argument is acceptable, then we should not reject the assumption that in the mutual interaction between nature and human activities, there must be something other than the facts that are accessible by scientific methods.

Thus, the assertion, shown in the preceding chapter, that the PP turns out to be invalid once we consider the problem of ignorance, is a conclusion based on two reasons derived from scientific and logical viewpoints: (1) the lack of scientific cause-effect relations, (2) no extension of the unexpected outcome. However, this conclusion is valid only in the fields of science and logic, which negatively connect the PP and the unexpected outcomes classified as ignorance. If this is the case, then it leaves some scope for considering ignorance beyond these restricted viewpoints, for, as Walker et al. say, "we have no way of knowing the full extent of our ignorance."

Accordingly, on the one hand, so far as we deal with unexpected outcomes along with scientific methods, we need to exclude ignorance as an irrational concept, although, as mentioned above, the PP does not function thoroughly without taking ignorance into consideration. However, if we renounce taking actions on the basis of science to deal with ignorance, we are forced to face the influx of unlimited imaginations, as suggested by Manson and Comstock, which is to be avoided. Then what should we do to protect the environment while duly considering ignorance and simultaneously complying with science and logic?

If we shift our attention from scrutinizing the potential hazard of each technological product to that of science in general, the problem might be avoided. In this case, the question should be modified: "Is the use of science/technology as a whole valid?"

When it comes to dealing with science in general, scientific knowledge is no longer a necessary condition, for the cause-effect relations between the use of science/technology as a whole and environmental degradation can be verified by our collective experience and historical facts. We can easily confirm that we had no environmental problems before the age of science/technology, and that the former has become even worse as the latter has developed. This is so obvious that we do not need to insist on scientific methods to confirm it. Interestingly, here we remain in the realm of science, relying on scientific methods, although in a very unsophisticated manner. We do not reject scientific methods, and we can still conclude that the development of science/technology has caused environmental degradation, and that the PP can be applied to science/technology as a whole. Furthermore, since we are questioning the danger of science in general, we do not need to focus our attention on individual technologies. This exempts us from having to categorize both uncertainty and ignorance, while we can still address the state of ignorance.

\section{Conclusion}

The general arguments that criticize the application of the PP to environmental problems do not investigate the nature of science/technology as a whole. Hence, RA remains a prerequisite as a scientific basis for implementing the PP, and arguments concerning unexpected outcomes that are derived from ignorance are neglected. However, this paper mentions that the application of the PP based on scientific methods is, in certain circumstances, useless because of incompetence in scientific methods while handling ignorance ${ }^{25}$. This strongly suggests that there exist certain defects in criticism based on scientific methods, but not in the conventional PP itself. 
Admittedly, we can inveigh against the ambiguous definition of the conventional PP when we accept the scientific viewpoint. However, this viewpoint depends on a scientific authenticity maintained by excluding ignorance, and therefore does not cover the original range of the PP that includes states of ignorance ${ }^{26)}$. Hence, we can conclude that criticism based on scientific methods has no capacity to deal with environmental problems.

Given all this, the reasoning suggested by Gardiner, that scientific evidence for threats is indispensable to avoiding the arbitral use of the PP does not ensure the proper use of the PP, and hence is no more than a political or temporary expedient. This sort of expedient may indeed lead us to a positive and practical argument and exempt us from a fruitless discussion of the so-called environmental zero-sum game. Nevertheless, it cannot play a crucial part in environmental protection activities unless the problem of ignorance is properly considered. Those who criticize the discussion of ignorance in the field of environmental protection measures as a zero-sum game, and those who prefer a practical argument that is based in the realm of science/technology and never go beyond it, have, in my opinion, no theoretical basis for asserting that their protection measures are logically sound and wholesome.

\section{Notes and References}

1) Readers can find the further explanation of this rather too absolute claim in the last paragraph of 6 th chapter.

2) Wingspread Conference on the Precautionary Principle, The Wingspread Consensus Statement on the Precautionary Principle (1998); available at: http://www.sehn.org/wing.html (accessed July 1, 2014).

3) "The fatal irony, into which scientific-technical society plunges us is, as a consequence of its perfection, much more radical: we do not know what it is we don't know-but from this dangers arise, which threaten mankind!" (U. Beck, "Living in the World Risk Society", Economy and Society, 35(3), 329-345 (2006).)

4) The possible methods for restricting the PP to the scope of scientific and cognitive are suggested by Gardiner, Saner and the European Environmental Agency as referred in Chapter 4.

5) C. Raffensberger and J. Tickner, eds., Protecting Public Health and the Environment: Implementing the Precautionary Principle, Washington, D.C.: Island Press (1999).

6) European Environment Agency, "Late Lessons from Early Warnings: the Precautionary Principle 1896-2000", Environmental Issue Report, 22 (2001).

7) RA and CBA still have important roles when dangers can be expressed as probabilities, though they are totally incapable of dealing with the outcomes categorized within the state of uncertainty and ignorance.

8) H. van den Belt, "Debating the Precautionary Principle: "Guilty until Proven Innocent" or "Innocent until Proven Guilty"?", Plant Physiology, 132, 1122-1126 (2003).

9) A.B. de González and S. Darby, "Risk of Cancer from Diagnostic X-rays: Estimates for the UK and 14 Other Countries", The Lancet, 363, 345-351 (2004).

10) H. Nishitani, "Current Topics on Radiation Exposure to Patients (Medical Exposure)", Shikoku Acta Medica, 62 (5-6) (2006).

11) J. Harris and S. Holm, "Extending Human Lifespan and the Precautionary Paradox", Journal of Medicine and Philosophy, 27 (3), 355-368 (2002).

12) G. Comstock, "Are the Policy Implications of the Precautionary Principle Coherent?, Talk at Harvard University", AgBioView Newsletter on Agricultural Biotechnology, (2000).

13) C.R. Sunstein, Laws of Fear: Beyond the Precautionary Principle, Cambridge University Press, p.14 (2005).

14) N.A. Manson, "Formulating the Precautionary Principle", Environmental Ethics, 24(3), 263-274 (2002).

15) N.A. Manson, "The Precautionay Principle, the Catastrophe Augument, and Pascal's Wager", The Journal of Scottish Philosophy, 4 (1) (1999).

16) S.M. Gardiner, "A Core Precautionary Principle", The Journal of Political Philosophy, 14 (1), 33-60 (2006).

17) A similar account of the need for scientific evidence for the proper use of the PP can be found in Ahteensuu's argument. In his view, "In sum, the argument from incoherence put forward by the critics of the PP is not convincing. The principle per se is not incoherent, and-given certain specifications to the trigger condition and to the prescribed action in the formation of the principle - it does not imply contradictory conduct-guidance. The critics seem to derive from a common source, namely from the consequences of our imperfect knowledge about the nature and its causal relationships and (sometimes stochastic) interactions." (M. Ahteensuu, "Defending the Precautionary Principle against Three Criticisms", Trames: A Journal of the Humanities \& Social Sciences, 11 (4), 366-381 (2007).)

18) M.A. Saner, "An Ethical Analysis of the Precautionary Principle", International Journal of Biotechnology, 4 (1), 81-95 (2002).

19) "Measures based on the precautionary principle 
must not be disproportionate to the desired level of protection and must not aim at zero risk, something which rarely exists." (Europian Commission, Communication from the Commission on the Precautionary Principle (2000).)

20) M.B.A. van Asselt and E. Vos, Perspectives on Uncertainty and Risk, Kluwer Academic Publishers (2000).

21) D.A. Schön and M. Rein, Frame Reflection: Toward the Resolution of Intractable Policy Controversies. Basic Books (1995).

22) A view like chaos theory, which understands the environment as a complex, nonequilibrium ecological system, presupposes dynamic systems of nature that are deterministic, i.e., their future behavior is fully determined by their initial conditions, although they yield widely diverging outcomes, rendering long-term prediction impossible in general. On such grounds, this view confines nature within a framework of physics, and hence does not deal with the ignorance discussed here.

23) W.E. Walker, P. Harremoës, J. Rotmans, J.P. van Der Sluijs, M.B.A. van Asselt, P. Janssen and M.P. Krayer von Krauss, "Defining Uncertainty: A Conceptual Basis for Uncertainty Management in Model-Based Decision Support", Integrated Assessment, 4 (1), 5-17 (2003).

24) Scientific causal relations are observed and confirmed only when we can apply scientific methods to the phenomenon in question.

25) As the idea of ignorance implies, so far as we insist on applying the PP, it is impossible to illustrate actual and concrete examples of ignorance. We cannot foresee and prepare for something we do not know what we do not know.

26) When the original range of the PP is concerned, it will be impossible to establish an acceptable definition or a set of criteria for the PP because, for example in its most simple and understandable form, the problems (A) to (C) suggested in Chapter 3 reappears as soon as we apply the PP to ignorance. 